NAPOLI, I8 - 26 MAGGIO I956

\title{
Second World Congress on Fertility and Sterility
}

\section{SGIENTIFIG PROGRAM}

\section{Section I}

Endocrine and Methabolic Factors in Fertility and Sterility. Iosé Botella-Llusia: Esterilidad por insuficiencia del cuerpo amarillo $y$ por defectos en las primeras etapas de la implantación; etc.

Section II

Professional Occupational and Toxic Factors in relation with Fertility. Theodor Bruck: Der Einfluss der Frauenarbeit und des Frauensports auf die weibliche Sterilität; etc.

Section III

New methods on diagnosis and treatment of male Sterility. Richard Hammen: Elektromikroscopische Untersuchungen der humanen Spermien;

Armando Trabucco, Belisario Otamendi, Luraschi: Determinación y dosaje de antigonadotropinas en los oligozoospermicos; etc.

Section IV

New methods on diagnosis and treatment of female Sterility.

I. Halbrecht: Latent Genital Tubercolosis as main Cause of Tubal Occlusion in primary Sterility; its Diagnosis and Treatment; etc. 


\title{
$I^{\circ}$ Congresso Internazionale di Genetica Medica
}

\section{(Copenhagen, I-6 agosto I956)}

\section{SVOLGIMENTO DEI L A VOR I}

I lavori del Congresso avranno luogo all'Istituto di Anatomia Medica di Copenhagen. In mattinata si terranno sedute plenarie, dedicate ai seguenti argomenti:

\author{
I agosto - Le mutazioni nell'uomo; \\ Radiazioni genetiche e implicazioni umanc. \\ 2 agosto - Ereditarietà quantitativa nell'uomo; \\ Patologia comparata e sperimentale in relazione alla gene- \\ tica umana; \\ Biochimica genetica nell'uomo. \\ 3 agosto - Metodi della genetica umana: a) lo studio della famiglia, \\ b) lo studio dei gemelli. \\ 4 agosto - Ricerche mediche e genetiche; \\ Applicazioni mediche della genetica umana. \\ 6 agosto - Applicazioni sociali della genetica medica; \\ Controllo epidemiologico delle malattie creditarie: I. Regi- \\ strazione genetico-igienica; 2. Accertamenti medico-genetici.
}

Nei pomeriggi dal $\mathrm{I}^{\circ}$ al 4 agosto si svolgeranno sedute di sezione, dedicate ai seguenti argomenti:

I. Genetica della popolazione, demografia c analisi statistica;

2. Ereditarietà multifattoriale nell'uomo;

3. Genetica biochimica nell'uomo;

4. Genetica antropologica (compresi i problemi di identificazionc, di parentela e lo sviluppo fisico);

5. Genetica citologica e radiazioni nell'uomo. Cromosomi umani;

6. Gruppi sanguigni e immuno-genetica nell'uomo. "Crossing-over " e "linkage";

7. Genetica e psichiatria, psicologia e criminologia;

8. Genetica e deficienze mentali;

9. Genetica e neurologia;

I0. Genetica e oftalmologia;

I I. Genetica e otolaringologia, difetti della parola;

I2. Genetica e odontologia;

13. Genetica e ortopedia, chirurgia e ginecologia;

I 4. Genetica e cancro;

I 5. Genetica e dermatologia;

16. Genetica e medicina interna;

I7. Genetica e medicina sociale.

\section{PRESENTAZIONE DI COMUNICAZIONI}

I partecipanti al Congresso potranno presentare una o più comunicazioni originali, da svolgere in non più di 15 minuti. Il riassunto delle comunicazioni, in una delle tre lingue ufficiali, dovrà essere inviato in tre copie dattiloscritte entro il I $^{\circ}$ aprile $195^{6}$. Esso non dovrà superare le 200 parole, compresi nome, titoli accademic: e titolo delle comunicazioni. Nei riassunti non dovranno essere comprese tabelle o formule di struttura; l'esposizione delle comunicazioni può essere illustrata con proiezioni di lastre e films. 